\title{
Geographic distribution of amino acid mutations in DHFR and DHPS in Plasmodium vivax isolates from Lao PDR, India and Colombia
}

\author{
Naowarat Saralamba ${ }^{1,2}$, Supatchara Nakeesathit ${ }^{2}$, Mayfong Mayxay ${ }^{3,4,5}$, Paul N. Newton ${ }^{3,5}$, Lyda Osorio ${ }^{6}$, \\ Jung-Ryong Kim ${ }^{7}$, Nicholas J. White ${ }^{2,5}$, Nicholas P. J. Day ${ }^{2,5}$, Arjen M. Dondorp ${ }^{2,5}$ and Mallika Imwong 1,2*
}

\begin{abstract}
Background: Non-synonymous mutations in dhfr and dhps genes in Plasmodium vivax are associated with sulfadoxine-pyrimethamine (SP) resistance. The present study aimed to assess the prevalence of point mutations in $P$. vivax dhfr (pvdhfr) and P. vivax dhps (pvdhps) genes in three countries: Lao PDR, India and Colombia.

Methods: Samples from 203 microscopically diagnosed vivax malaria were collected from the three countries. Five codons at positions 13,57,58,61, and 117 of pvdhfr and two codons at positions 383 and 553 of pvdhps were examined by polymerase chain reaction-restriction fragment length polymorphism methodology.

Results: The largest number of 58R/117 N double mutations in pvdhfr was observed in Colombia (94.3\%), while the corresponding wild-type amino acids were found at high frequencies in Lao PDR during 2001-2004 (57.8\%). Size polymorphism analysis of the tandem repeats within pvdhfr revealed that $74.3 \%$ of all the isolates carried the type B variant. Eighty-nine per cent of all the isolates examined carried wild-type pvdhps A383 and A553.

Conclusions: Although SP is not generally used to treat $P$. vivax infections, mutations in $d h f r$ and $d h p s$ that confer antifolate resistance in P. vivax are common. The data strongly suggest that, when used primarily to treat falciparum malaria, SP can exert a substantial selective pressure on P. vivax populations, and this can lead to point mutations in $d h f r$ and dhps. Accurate data on the global geographic distribution of $d h f r$ and $d h p s$ genotypes should help to inform anti-malarial drug-use policies.
\end{abstract}

Keywords: Plasmodium vivax, Sulfadoxine-pyrimethamine, dhfr, dhps

\section{Background}

The antifolate drug combination sulfadoxine-pyrimethamine (SP) has been used as an anti-malarial treatment because of its low cost and relative safety. The therapeutic targets of SP in malaria parasites are dihydropteroate synthase (DHPS) and dihydrofolate reductase (DHFR) enzymes. Molecular epidemiology studies have revealed that the point mutations in the malaria parasite's $d h f r$ and dhps genes, which confer resistance to SP, change the amino acid residues around the active sites of the

\footnotetext{
*Correspondence: mallika.imw@mahidol.ac.th

1 Department of Molecular Tropical Medicine and Genetics, Faculty

of Tropical Medicine, Mahidol University, Bangkok, Thailand

Full list of author information is available at the end of the article
}

enzymes they encode [1]. Progressive accumulation of point mutations is associated with increasing levels of drug resistance in malaria parasites. In Plasmodium falciparum, a mutation at codon 108 of DHFR, which confers low-grade pyrimethamine resistance, is observed first, followed by additional mutations that induce high-level pyrimethamine resistance at codons 51 and 59 [2]. Accumulation of $P$. falciparum DHFR (PfDHFR) mutations at codons $16,51,59,108$, and 164 is associated with progressively increasing levels of pyrimethamine resistance [3]. Patients infected with $P$. falciparum isolates carrying these point mutations are more likely to experience SP treatment failure $[4,5]$. Point mutations have been identified in Plasmodium vivax DHFR (PvDHFR) at codons 51, 58 and 117, which correspond to PfDHFR positions 
51, 59 and 108 [6-8]. Additionally, PvDHFR mutations at codons 57 and 61 in combination with those at codons 58 and 117 have been reported to correlate with SP treatment failure $[9,10]$.

Point mutations in PfDHPS codons 436, 437, 540, 581, and 613 have been found to be associated with sulfadoxine resistance [11], and in P. vivax homologous PvDHPS mutations have been described at codons $382,383,512$, 553, and 585. Mutations in PvDHPS codon 383 and 553, which correspond to PfDHPS positions 437 and 581, have been found at high prevalence in Thailand [12, 13]. Parasites carrying multiple mutations in $p v d h f r$ and pvdhps are cleared more slowly from the patient's blood during SP treatment [12]. Because monitoring of antimalarial drug susceptibility in $P$. vivax parasites in vitro is limited by the difficulties in culturing this parasite species, molecular markers for SP resistance are an important tool for evaluating SP resistance patterns in $P$. vivax populations. The well-described patterns of mutations related to drug resistance in $P$. falciparum and $P$. vivax have led to the development of the polymerase chain reaction-restriction fragment length polymorphism (PCR-RFLP) technique as a molecular surveillance tool for predicting SP drug resistance in specific geographical areas.

Molecular studies on point mutations in $p v d h f r$ and pvdhps have been reported in many countries. Mutations in $p v d h f r$ that confer SP resistance are found to be distributed wildly in Thailand [7, 13, 14], Myanmar [15], Cambodia [16], Vietnam [17], Indonesia [18, 19], Papua New Guinea [20], the Philippines [21], Bangladesh [21], Nepal [21], Pakistan [22], China [23], and India [8, 24, 25]. Although data on $p v d h f r$ and $p v d h p s$ genotypes are available for many Southeast Asian countries, such data remain limited in some $P$. vivax-endemic areas, notably Lao PDR and South America. Reports from South America have shown that high prevalence of PvDHFR double mutations in codon 58 and 117 were found in Colombia [26, 27] while most of the samples from French Guiana have multiple point mutations in PvDHFR [28]. In this study, the prevalences of point mutations in $p v d-$ $h f r$ and $p v d h p s$ were studied in three countries: Lao PDR, Colombia and India. India and Colombia are two countries in which $P$. vivax is present as the predominant malaria infection-causing species [29-31]. The prevalence of antifolate anti-malarial drug resistance in India is relatively high, with many recent reports of mutations in $p v d h f r$ and pvdhps, whereas information on drug resistance mutations in Colombia remains limited. Plasmodium vivax infections are common in rural Laos [32], but no published information is available on $p v d h f r$ and pvdhps mutations.
In this study, point mutations in $p v d h f r$ and $p v d h p s$ were investigated as a tool for surveillance of SP resistance. The pattern of mutations present in each study area provide valuable molecular information on antifolate drug resistance, and this information may be useful for epidemiological mapping of drug-resistant vivax malaria.

\section{Methods \\ Parasite isolates and DNA extraction}

Blood samples were collected from symptomatic $P$. vivax-infected patients as either whole blood or dried blood spots obtained from three different countries: Lao PDR, India and Colombia. Plasmodium vivax isolates $(\mathrm{n}=136)$ from Lao PDR were collected between 2001 and 2004, and 45 samples were collected between 2008 and 2012. In India, the 117 samples were collected from patients who attended the malaria clinic at the Calcutta School of Tropical Medicine, Kolkata between April 2003 and September 2004. Fifty-three P. vivax isolates from Colombia were collected between 2001 and 2004 from five areas (Amazonas, Buenaventuno, Red Tumaco, Red Equapi, and Quibdó). All of the clinical isolates from the three countries were reported as single-species infections of $P$. vivax as determined by light microscopic examination of Giemsa-stained blood smears. This study received ethical approval from the Faculty of Tropical Medicine, Mahidol University (MUTM 2011-055-01).

Genomic DNA was extracted from all the isolates using a commercially available DNA extraction kit (QIAGEN, Germany) following the manufacturer's instructions. DNA was extracted from $200 \mu \mathrm{l}$ of whole blood or one dried blood spot on Whatman 3MM filter paper $(1 \mathrm{~cm}$ diameter) in a final elution volume of $100 \mu \mathrm{l}$. DNA samples were kept at $-20^{\circ} \mathrm{C}$ before use.

\section{PCR analysis of parasite species}

Confirmation of the microscopic detection of $P$. vivax and detection of other cryptic species that might be in the samples were achieved using a nested PCR amplification assay based on the SSU rRNA gene [33].

\section{PCR-RFLP of PvDHFR and PvDHPS}

Nested or semi-nested PCR amplification of PvDHFR was carried out using a method described previously [8]. Five point mutations (I13L, F57I/L, S58R, T61 M, S117 N/T) were examined by RFLP using the corresponding restriction enzymes for each specific position in the pvdhfr gene [8]. In the case of PvDHPS, two positions (A383G and A553G) were investigated by PCRRFLP, following the protocol described previously [12]. All the DNA fragments obtained from the RFLP analysis were subjected to electrophoresis on $2 \%$ agarose gels 
before visualization on an ultraviolet transilluminator after ethidium bromide staining.

\section{Detection of size polymorphisms in PvDHFR}

The tandem repeat region between nucleotides 262 and 309 of the pvdhfr gene was PCR amplified using a protocol published previously [7]. Allelic size variants, which were assigned as described previously [7], were designated A, B and C from the largest to the smallest in base pairs, respectively (range, ca. 230-280 bp).

\section{Results}

Of the 203 P. vivax malaria samples from the three countries, nested PCR detected nine samples containing mixed infections with $P$. falciparum. Mixed infections were present at high frequency in Lao PDR (2001-2004, eight out of 98 samples), while in India mixed infections accounted for one of 32 samples, and there were no mixed infections in the 28 Colombian samples. The result have shown demographic history of vivax population and their resistant background in each region, which can be part of further consideration for malaria eradication programme.
Genotype polymorphisms within PvDHFR were examined in five codons at positions 13,57, 58, 61, and 117 (Fig. 1). Most of the samples collected from Lao PDR during 2001-2004 (89 out of 154) contained wild-type sequences at these five codons, compared with the $58 \mathrm{R}$ and $117 \mathrm{~N}$ double mutations found at high frequencies during 2008-2012 (29 out of 45 samples). High numbers of double mutations were also found within Colombia (51 out of 53) and India (45 out of 111). The number of haplotypes based on these five residues in PvDHFR was limited for Colombia, India and Lao PDR during 2008-2012 in comparison with the samples from Lao PDR during 2001-2004. The total numbers of PvDHFR haplotypes for Colombia, India and Lao PDR during 2008-2012 were three, five and three, respectively. The majority of the non-wild-type genotypes identified in all three regions had double mutations at codons 58R and $117 \mathrm{~N}$. Double mutations in $58 \mathrm{R} / 117 \mathrm{~N}$ were found at high frequency in the Colombian isolates (51 out of 53 samples) and in the Lao PDR isolates between 2008 and 2012 (29 out of 45 samples). It was noticed that mixed genotype infections of wild-type and mutant parasites were found at high frequency in the isolates from Lao PDR (2001-2004, 26 of

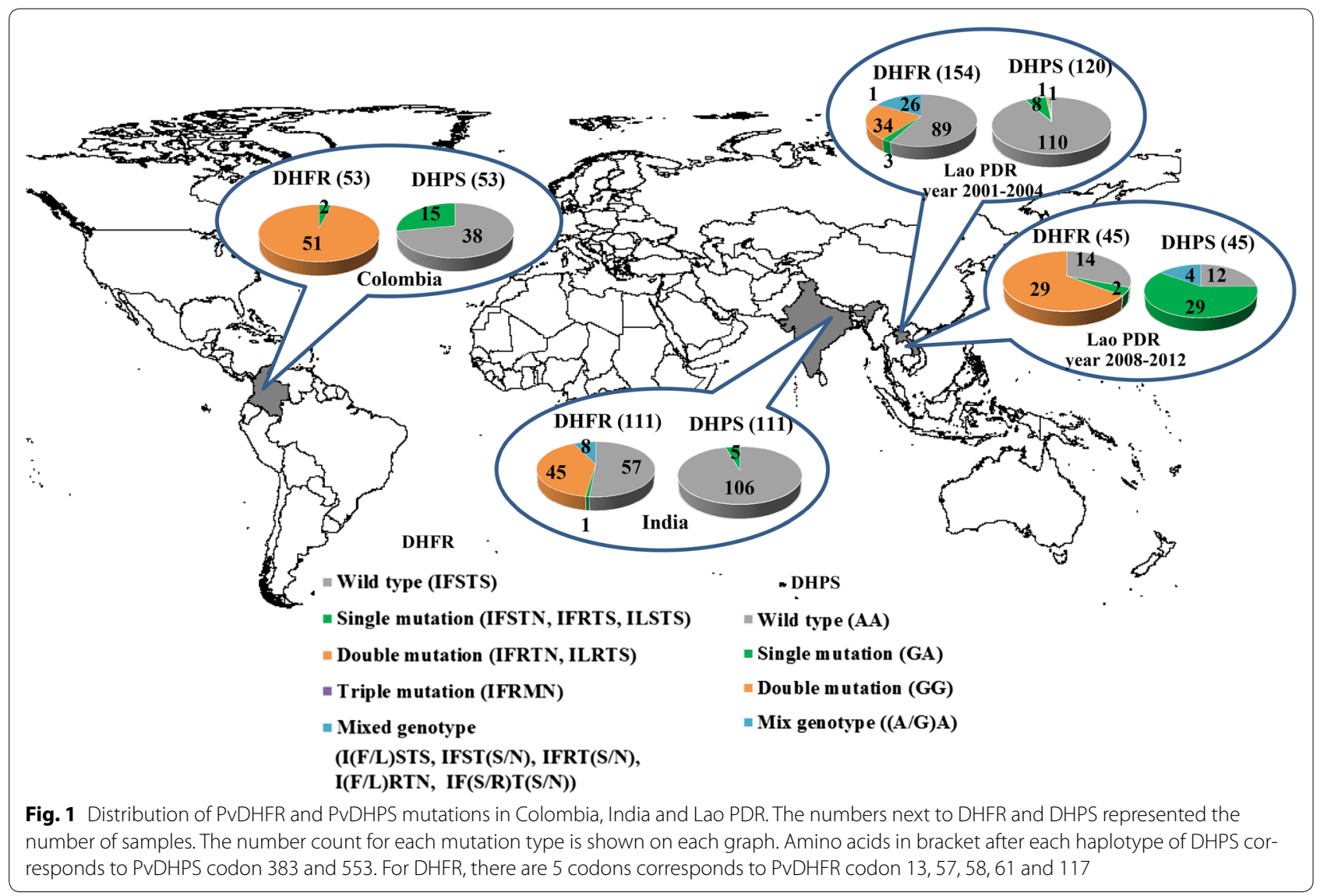


154), while there were no mixed genotype infections in Colombia.

Size polymorphisms in $p v d h f r$ were investigated in 48 , 92, 45, and 117 samples from Colombia, Lao PDR (20012004), Lao PDR (2008-2012) and India, respectively. The samples from all three countries contained a high prevalence of the parasite allelic variant type $B$, except Lao PDR (2008-2012), which had a high frequency of the allelic type $\mathrm{C}$. No mixed allelic types were found in India and Lao PDR (2008-2012), but mixed A/B and A/C allelic types were found in seven and six samples, respectively, from Lao PDR (2001-2004), while one and two samples each of mixed allelic types, $\mathrm{A} / \mathrm{B}$ and $\mathrm{A} / \mathrm{C}$, respectively, were identified in the Colombian samples (Fig. 2). Part of samples that carry allelic variant type A, B, and $\mathrm{C}$ with PCR product size approximating 280,250 , and $230 \mathrm{bp}$, and their sequences around the central repeat unit were subsequently determined. Deduced amino acid sequences of type A, B, and C are GGDNTS GGDNTH GGDNTH GGDNAD, GGDNTS GGDNTH GGDNAD, and GGDNTS GGDNAD, respectively.

Point mutations in PvDHPS were investigated at two positions: A383G and A553G. The majority of the samples from all three countries contained wild-type residues at these two positions (Fig. 1). The 383G single mutation was found in all three countries at frequencies of $28.3 \%$ (Colombia), 6.7 \% (Lao PDR, 2001-2004), 64.4 \% (Lao
PDR, 2008-2012) and $4.5 \%$ (India). Since the mutation at $383 \mathrm{G}$ was often found with $382 \mathrm{~S}$ in Colombia and associated with SP resistance [27], eleven samples carrying $383 \mathrm{G}$ and 9 wild type samples were submitted for sequencing. It was found that all 20 samples carry wild type amino acid at codon 382. Double mutations at 383G and 553G and mixed genotypes of the wild-type and the position 383 mutation were found in one sample for each type within the samples from Lao PDR (2001-2004).

Haplotype classification based on five point mutations in PvDHFR and two point mutations in PvDHPS was achievable for 53, 109, 45, and 111 samples from Colombia, Lao PDR (2001-2004), Lao PDR (2008-2012) and India, respectively. Figure 3 shows the number of haplotypes identified in samples from each country for the 15 haplotypes identified herein. Samples from Lao PDR (2001-2004) and India showed a high prevalence of the wild-type IFSTSAA haplotype at 60.6 and $49.5 \%$, respectively. A double point mutation (58R/117 N) in PvDHFR in combination with wild-type PvDHPS IFRTNAA was found with high frequency in Colombia at $69.8 \%$. Comparing the two time periods for Lao PDR revealed that the period 2008-2012 contained a high frequency of mutant alleles. It was found that the double $58 \mathrm{R} / 117 \mathrm{~N}$ mutation in PvDHFR and the single 383G mutation in DHPS predominated in Lao PDR between 2008 and 2012, with a value of $31.1 \%$.

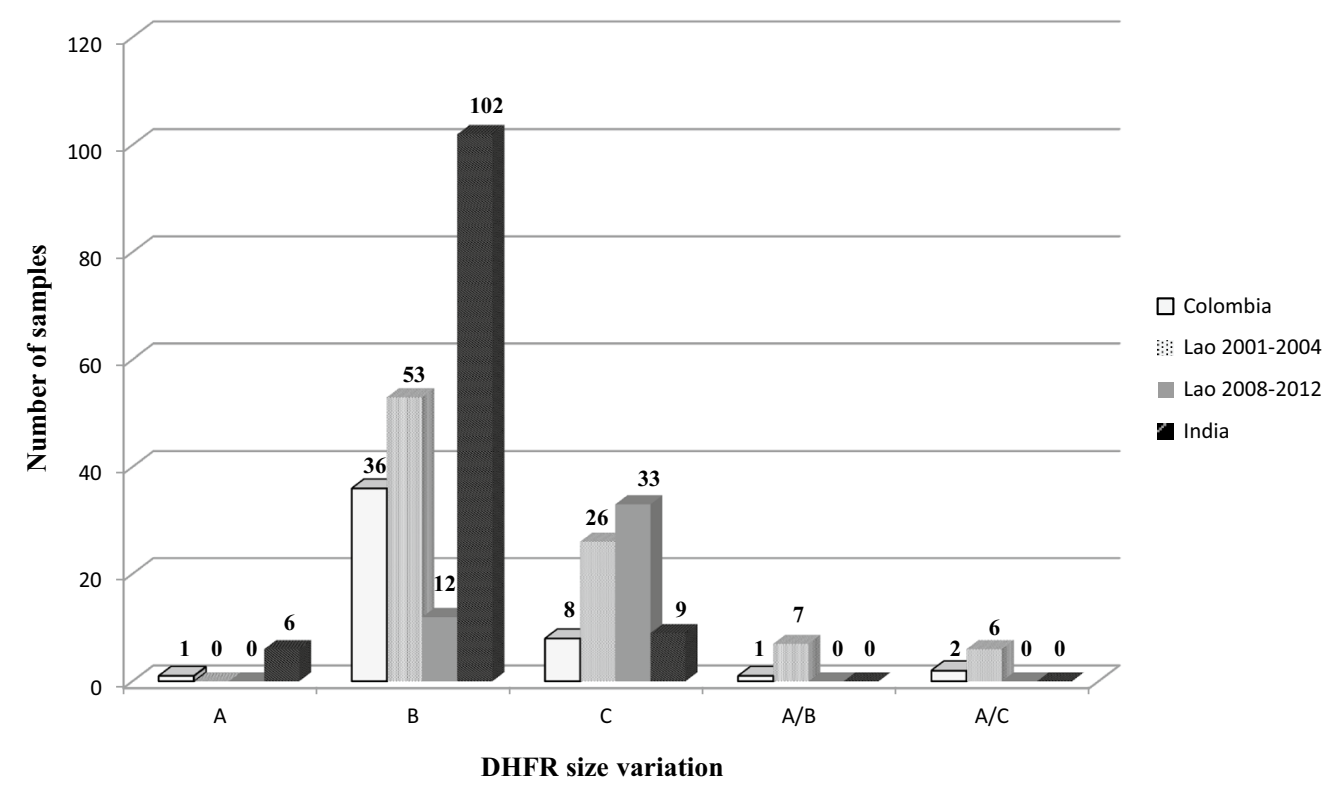

Fig. 2 Distribution of DHFR allelic size variations in Plasmodium vivax parasites from Colombia, India and Lao PDR, based on the tandem repeats within pvdhfr. The numbers on top of each graph represent the number of samples. Capital letters on the $x$-axis represent the allelic types 


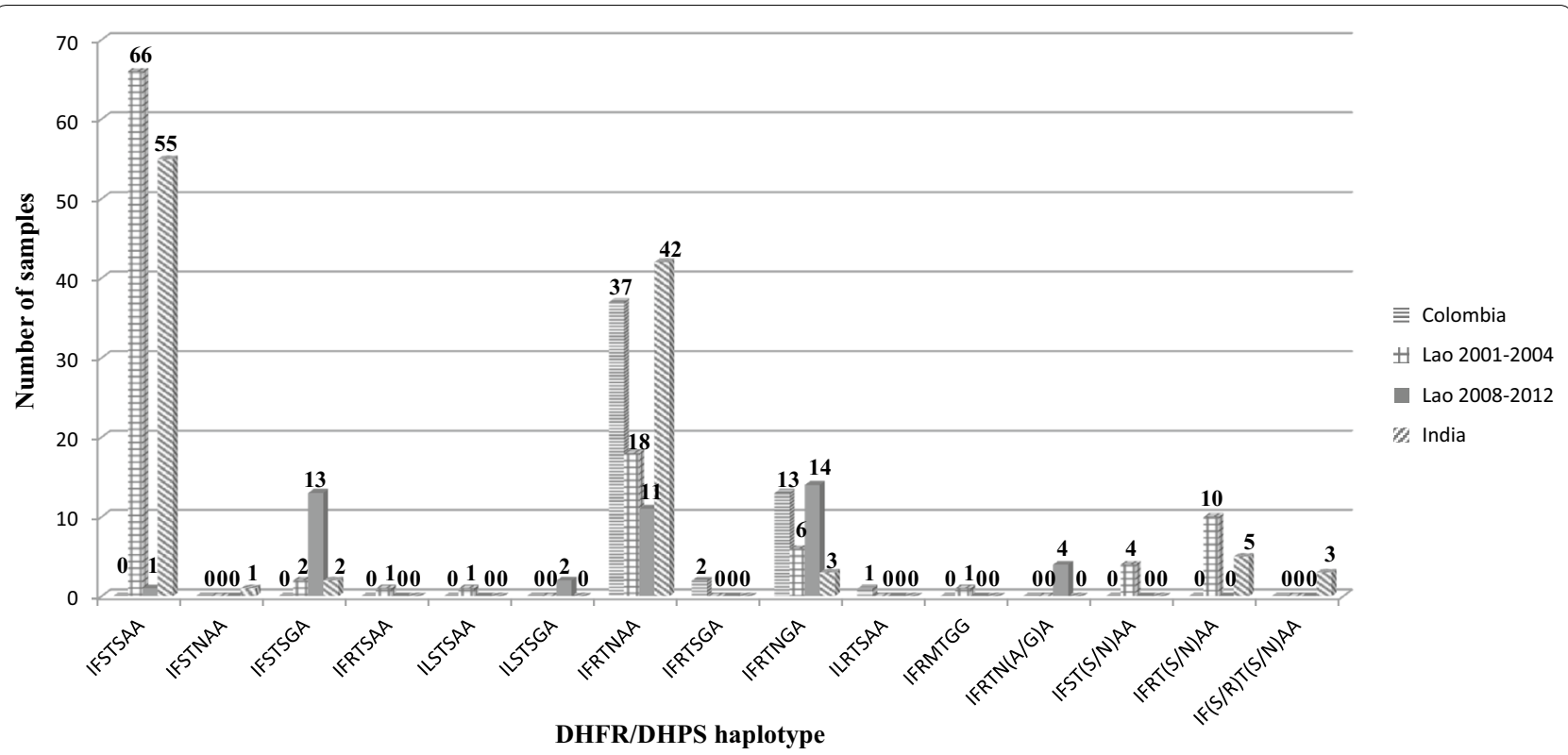

Fig. 3 Distribution of DHFR-DHPS haplotypes found in Colombia, India and Lao PDR. The numbers on top of each graph represent the number of samples. The $x$-axis shows the various haplotypes. The amino acid sequence for each haplotype corresponds to PvDHFR codons 13, 57, 58, 61, 117, PvDHPS codons 383 and 553

\section{Discussion}

In this study, the distribution of mutant alleles in $p v d h f r$ and $p v d h p s$ was examined in three geographical regions with different levels of $P$. vivax endemicity and differing histories of recent drug usage. India and Colombia have a relatively high prevalence of $P$. vivax infections (60-65\%) compared with $P$. falciparum infections [30, 31, 34], while Lao PDR has a relatively low prevalence of $P$. vivax infections (20-25\%) compared with $P$. falciparum infections [32]. Perhaps because of this, the prevalence of cryptic $P$. falciparum co-infections in patients presenting with $P$. vivax infections in Lao PDR has been reported to be $10 \%$ [35], a figure consistent with the data from the current study.

Size polymorphism regarding the GGDN repeat unit in PvDHFR was investigated and the result showed high prevalence of allelic variant type B in most of the samples. The patterns of PvDHFR point mutations in each allelic type were considered and the result showed no significant association. This result was similar to the previous findings where PvDHFR point mutations did not show significant association to any allelic type $[6,16,19]$. As shown from the crystal structure, the GGDN repeat region was located outside the binding pocket of PvDHFR enzyme and thus thought to be nonessential for substrate binding [36].

Surveillance of SP resistance was investigated with PCR-RFLP to determine specific point mutation in five codons of PvDHFR and two codons of PvDHPS. This information provides some points of view for epidemiological mapping of SP resistance and their background situations in vivax malaria. Plasmodium vivax parasites from India were found to have a high frequency of $d h f r$ and dhps mutations [37]. Recent studies of PvDHFR genotypes in India have reported high frequencies of $58 \mathrm{R}$ and $117 \mathrm{~N}$ double mutations [37], which is similar to the results for PfDHFR mutations $[38,39]$. The results from this study showed that $P$. vivax parasites from India carried the wild-type DHFR allele at approximately the same high frequency $(51.4 \%$ ) as they carried the $58 \mathrm{R} / 117 \mathrm{~N}$ double mutation (40.5\%), suggesting that the double mutation and wild-type alleles are still common in India. SP is not the drug of choice for treatment of $P$. vivax infections in India because $P$. vivax is still susceptible to chloroquine [30]. Therefore, the appearance of the double mutation in PvDHFR is likely to be caused by the use of SP to treat $P$. falciparum infections, thereby exerting a drug selection pressure on the sympatric $P$. vivax population. The data suggest that use of antifolate combinations for $P$. vivax infections should be carefully validated. The therapeutic efficacy of the SP drug combination should be investigated.

Analysis of the pvdhfr mutations in Colombia revealed a high frequency of the $58 \mathrm{R}$ and $117 \mathrm{~N}$ double mutation, similar to previous observations [27]; this double mutation in DHFR was also found at high frequency in $P$. falciparum [40]. It has been proposed that use of SP in Colombia has exerted an evolutionary selection pressure 
on both $P$. falciparum and $P$. vivax. Therefore, the SP combination must be carefully validated for its efficacy.

No scientific data for $p v d h f r$ mutations in Lao PDR have been published. Infections caused by $P$. falciparum, the predominant malaria parasite species in Lao PDR, were found to contain double and triple mutations in DHFR [41-43]. This survey in Lao PDR encapsulated two time periods: 2001-2004 and 2008-2012. From 2001-2004, most of the $P$. vivax population still carried the wild-type allele for $d h f r$. The number of mutations increased and double mutations were found in $p v d-$ $h f r$, while a single mutation was found in $p v d h p s$ during 2008-2012. As $P$. vivax is often found to co-exist with the more prevalent $P$. falciparum in Laos, it is likely that $P$. vivax has been under heavy SP pressure before the switch to artemisinin-based combination therapy (ACT). It has been speculated that the change in treatment policy to ACT was timely. However, artemisinin resistance has already emerged and spread in Southeast Asia. Epidemiological surveys aimed at identifying artemisinin-resistant parasites in Lao PDR are essential for assessing the impact of ACT policy in this country.

\section{Conclusion}

These data suggest strongly that SP used to treat $P$. falciparum exerts a substantial selective pressure on the $P$. vivax population, leading to the selection of drug resistance-conferring mutations in $p v d h f r$ and $p v d h p s$.

\begin{abstract}
Abbreviations
ACT: artemisinin-based combination therapy; DHFR: dihydrofolate reductase; DHPS: dihydropteroate synthase; PCR-RFLP: polymerase chain reactionrestriction fragment length polymorphism; PVDHFR: Plasmodium vivax dihydrofolate reductase; PVDHPS: Plasmodium vivax dihydropteroate synthase; SP: sulfadoxine-pyrimethamine.
\end{abstract}

\section{Authors' contributions}

NS was involved in all stages of this study. MI supervised the molecular genetics study and SN the laboratory work. MM, PNN, LO, and JRK coordinated the samples used for the laboratory work. MI, NJW, NPD, and AMD helped to write the manuscript and provided constructive advice on its content. All authors read and approved the final manuscript.

\section{Author details \\ ${ }^{1}$ Department of Molecular Tropical Medicine and Genetics, Faculty of Tropical Medicine, Mahidol University, Bangkok, Thailand. ${ }^{2}$ Mahidol-Oxford Tropical Medicine Research Unit, Faculty of Tropical Medicine, Mahidol University, Bangkok, Thailand. ${ }^{3}$ Lao-Oxford-Mahosot Hospital-Wellcome Trust Research Unit (LOMWRU), Microbiology Laboratory, Mahosot Hospital, Vientiane, Lao People's Democratic Republic. ${ }^{4}$ Faculty of Postgraduate Studies, University of Health Sciences, Vientiane, Lao People's Democratic Republic. ${ }^{5}$ Cen- tre for Tropical Medicine and Global Health, Churchill Hospital, University of Oxford, Oxford, UK. ${ }^{6}$ International Centre for Medical Research and Training (CIDEIM), Cali, Colombia. ${ }^{7}$ Centre for Tropical Medicine \& Parasitology, Kolkata, India.}

\section{Acknowledgements}

We thank all the patients and staff for the samples from Colombia, and the staff and nurses at the Centre of Tropical Medicine and Parasitology, Kolkata, India for their help. We also wish to thank the Director and staff of the Centre for Malariology, Parasitology and Entomology, Vientiane, Savannakhet Provincial Malaria Station and Phalanxay District Hospital, Lao PDR. This study was financed in part by Mahidol University, Thailand, and was part of the Wellcome Trust Mahidol University-Oxford Tropical Medicine Research Programme supported by the Wellcome Trust of Great Britain.

\section{Competing interests}

The authors declare that they have no competing interests.

\section{Ethics approval and consent to participate}

This study received ethical approval from the Faculty of Tropical Medicine, Mahidol University (MUTM 2011-055-01).

\section{Funding}

This study was funded by Mahidol University, Thailand, and the Wellcome Trust Mahidol University-Oxford Tropical Medicine Research Programme supported by the Wellcome Trust of Great Britain.

Received: 14 June 2016 Accepted: 16 September 2016

Published online: 21 September 2016

\section{References}

1. Sirawaraporn W, Prapunwattana P, Sirawaraporn R, Yuthavong Y, Santi DV. The dihydrofolate reductase domain of Plasmodium falciparum thymidylate synthase-dihydrofolate reductase. Gene synthesis, expression, and anti-folate-resistant mutants. J Biol Chem. 1993;268:21637-44.

2. Sirawaraporn W, Sathitkul T, Sirawaraporn R, Yuthavong Y, Santi DV. Antifolate-resistant mutants of Plasmodium falciparum dihydrofolate reductase. Proc Natl Acad Sci. 1997:94:1124-9.

3. Sibley $\mathrm{CH}$, Hyde JE, Sims PF, Plowe CV, Kublin JG, Mberu EK, et al. Pyrimethamine-sulfadoxine resistance in Plasmodium falciparum: what next? Trends Parasitol. 2001;17:582-8.

4. Plowe CV, Cortese JF, Djimde A, Nwanyanwu OC, Watkins WM, Winstanley PA, et al. Mutations in Plasmodium falciparum dihydrofolate reductase and dihydropteroate synthase and epidemiologic patterns of pyrimethamine-sulfadoxine use and resistance. J Infect Dis. 1997;176:1590-6.

5. Mutabingwa TK, Maxwell CA, Sia IG, Msuya FH, Mkongewa S, Vannithone $\mathrm{S}$, et al. A trial of proguanil-dapsone in comparison with sulfadoxinepyrimethamine for the clearance of Plasmodium falciparum infections in Tanzania. Trans R Soc Trop Med Hyg. 2001;95:433-8.

6. de Pecoulas PE, Tahar R, Ouatas T, Mazabraud A, Basco LK. Sequence variations in the Plasmodium vivax dihydrofolate reductase-thymidylate synthase gene and their relationship with pyrimethamine resistance. Mol Biochem Parasitol. 1998;92:265-73.

7. Imwong M, Pukrittakayamee S, Looareesuwan S, Pasvol G, Poirreiz J, White NJ, et al. Association of genetic mutations in Plasmodium vivax dhfr with resistance to sulfadoxine-pyrimethamine: geographical and clinical correlates. Antimicrob Agents Chemother. 2001;45:3122-7.

8. Imwong M, Pukrittayakamee S, Renia L, Letourneur F, Charlieu JP, Leartsakulpanich $U$, et al. Novel point mutations in the dihydrofolate reductase gene of Plasmodium vivax: evidence for sequential selection by drug pressure. Antimicrob Agents Chemother. 2003;47:1514-21.

9. Hastings MD, Porter KM, Maguire JD, Susanti I, Kania W, Bangs MJ, et al. Dihydrofolate reductase mutations in Plasmodium vivax from Indonesia and therapeutic response to sulfadoxine plus pyrimethamine. J Infect Dis. 2004;189:744-50.

10. Tjitra E, Baker J, Suprianto S, Cheng Q, Anstey NM. Therapeutic efficacies of artesunate-sulfadoxine-pyrimethamine and chloroquine-sulfadoxinepyrimethamine in vivax malaria pilot studies: relationship to Plasmodium vivax dhfr mutations. Antimicrob Agents Chemother. 2002;46:3947-53.

11. Triglia T, Menting JG, Wilson C, Cowman AF. Mutations in dihydropteroate synthase are responsible for sulfone and sulfonamide resistance in Plasmodium falciparum. Proc Natl Acad Sci USA. 1997;94:13944-9.

12. Imwong M, Pukrittayakamee S, Cheng Q, Moore C, Looareesuwan $\mathrm{S}$, Snounou G, et al. Limited polymorphism in the dihydropteroate synthetase gene (dhps) of Plasmodium vivax isolates from Thailand. Antimicrob Agents Chemother. 2005;49:4393-5. 
13. Rungsihirunrat K, Sibley CH, Mungthin M, Na-Bangchang K. Geographical distribution of amino acid mutations in Plasmodium vivax DHFR and DHPS from malaria endemic areas of Thailand. Am J Trop Med Hyg. 2008;78:462-7.

14. Thongdee P, Kuesap J, Rungsihirunrat K, Tippawangkosol P, Mungthin M, Na-Bangchang K. Distribution of dihydrofolate reductase (dhfr) and dihydropteroate synthase (dhps) mutant alleles in Plasmodium vivax isolates from Thailand. Acta Trop. 2013;128:137-43.

15. Lu F, Lim CS, Nam DH, Kim K, Lin K, Kim TS, et al. Mutations in the antifolate-resistance-associated genes dihydrofolate reductase and dihydropteroate synthase in Plasmodium vivax isolates from malaria-endemic countries. Am J Trop Med Hyg. 2010;83:474-9.

16. de Pecoulas PE, Tahar R, Yi P, Thai KH, Basco LK. Genetic variation of the dihydrofolate reductase gene in Plasmodium vivax in Snoul, northeastern Cambodia. Acta Trop. 2004;92:1-6.

17. Auliff A, Wilson DW, Russell B, Gao Q, Chen N, le Anh N, et al. Amino acid mutations in Plasmodium vivax DHFR and DHPS from several geographical regions and susceptibility to antifolate drugs. Am J Trop Med Hyg. 2006;75:617-21.

18. Asih PB, Marantina SS, Nababan R, Lobo NF, Rozi IE, Sumarto W, et al. Distribution of Plasmodium vivax pvdhfr and pvdhps alleles and their association with sulfadoxine-pyrimethamine treatment outcomes in Indonesia. Malar J. 2015;14:365.

19. Hastings MD, Maguire JD, Bangs MJ, Zimmerman PA, Reeder JC, Baird $\mathrm{JK}$, et al. Novel Plasmodium vivax dhfr alleles from the Indonesian Archipelago and Papua New Guinea: association with pyrimethamine resistance determined by a Saccharomyces cerevisiae expression system. Antimicrob Agents Chemother. 2005;49:733-40.

20. Barnadas C, Timinao L, Javati S, Iga J, Malau E, Koepfli C, et al. Significant geographical differences in prevalence of mutations associated with Plasmodium falciparum and Plasmodium vivax drug resistance in two regions from Papua New Guinea. Malar J. 2015;14:399.

21. Thongdee P, Kuesap J, Rungsihirunrat K, Dumre SP, Espino E, Noedl $\mathrm{H}$, et al. Genetic polymorphisms in Plasmodium vivax dihydrofolate reductase and dihydropteroate synthase in isolates from the Philippines, Bangladesh, and Nepal. Korean J Parasitol. 2015;53:227-32.

22. Raza A, Ghanchi NK, Khan MS, Beg MA. Prevalence of drug resistance associated mutations in Plasmodium vivax against sulphadoxinepyrimethamine in southern Pakistan. Malar J. 2013;12:261.

23. Huang B, Huang S, Su XZ, Guo H, Xu Y, Xu F, et al. Genetic diversity of Plasmodium vivax population in Anhui province of China. Malar J. 2014;13:13.

24. Kaur S, Prajapati SK, Kalyanaraman K, Mohmmed A, Joshi H, Chauhan VS. Plasmodium vivax dihydrofolate reductase point mutations from the Indian subcontinent. Acta Trop. 2006;97:174-80.

25. Prajapati SK, Verma A, Adak T, Yadav RS, Kumar A, Eapen A, et al. Allelic dimorphism of Plasmodium vivax gam-1 in the Indian subcontinent. Malar J. 2006;5:90

26. Hawkins VN, Auliff A, Prajapati SK, Rungsihirunrat K, Hapuarachchi HC, Maestre A, et al. Multiple origins of resistance-conferring mutations in Plasmodium vivax dihydrofolate reductase. Malar J. 2008;7:72.

27. Hawkins VN, Joshi H, Rungsihirunrat K, Na-Bangchang K, Sibley CH. Antifolates can have a role in the treatment of Plasmodium vivax. Trends Parasitol. 2007;23:213-22.

28. Barnadas C, Musset L, Legrand E, Tichit M, Briolant S, Fusai T, et al. High prevalence and fixation of Plasmodium vivax dhfr/dhps mutations related to sulfadoxine/pyrimethamine resistance in French Guiana. Am J Trop Med Hyg. 2009;81:19-22.

29. Chaparro P, Padilla J, Vallejo AF, Herrera S. Characterization of a malaria outbreak in Colombia in 2010. Malar J. 2013;12:330.

30. Nandy A, Addy M, Maji AK, Bandyopadhyay AK. Monitoring the chloroquine sensitivity of Plasmodium vivax from Calcutta and Orissa, India. Ann Trop Med Parasitol. 2003;97:215-20.

31. Cristiano E, Patrucco L, Rojas Jl. A systematic review of the epidemiology of multiple sclerosis in South America. Eur J Neurol. 2008;15:1273-8.

32. Khaminsou N, Kritpetcharat O, Daduang J, Kritpetcharat P. A survey of malarial infection in endemic areas of Savannakhet province, Lao PDR and comparative diagnostic efficiencies of Giemsa staining, acridine orange staining, and semi-nested multiplex PCR. Parasitol Int. 2008;57:143-9.

33. Snounou G, Singh B. Nested PCR analysis of Plasmodium parasites. Methods Mol Med. 2002;72:189-203.

34. Maestre A, Sunil S, Ahmad G, Mohmmed A, Echeverri M, Corredor M, et al. Inter-allelic recombination in the Plasmodium vivax merozoite surface protein 1 gene among Indian and Colombian isolates. Malar J. 2004;3:4.

35. Mayxay M, Pukritrayakamee S, Chotivanich K, Imwong M, Looareesuwan S, White NJ. Identification of cryptic coinfection with Plasmodium falciparum in patients presenting with vivax malaria. Am J Trop Med Hyg. 2001;65:588-92.

36. Kongsaeree P, Khongsuk P, Leartsakulpanich U, Chitnumsub P, Tarnchompoo B, Walkinshaw MD, et al. Crystal structure of dihydrofolate reductase from Plasmodium vivax: pyrimethamine displacement linked with mutation-induced resistance. Proc Natl Acad Sci USA. 2005;102:13046-51.

37. Ganguly S, Saha P, Chatterjee M, Maji AK. Prevalence of polymorphisms in antifolate drug resistance molecular marker genes pvdhfr and pvdhps in clinical isolates of Plasmodium vivax from Kolkata, India. Antimicrob Agents Chemother. 2014;58:196-200.

38. Sharma J, Khan SA, Dutta P, Mahanta J. Molecular determination of antifolate resistance associated point mutations in Plasmodium falciparum dihydrofolate reductase (dhfr) and dihydropteroate synthetase (dhps) genes among the field samples in Arunachal Pradesh. J Vector Borne Dis. 2015;52:116-21.

39. Pathak A, Martensson A, Gawariker S, Mandliya J, Sharma A, Diwan V, et al. Characterization of drug resistance associated genetic polymorphisms among Plasmodium falciparum field isolates in Ujjain, Madhya Pradesh, India. Malar J. 2014;13:182.

40. Hernandez DC, Guerra AP, Cucunuba ZM, Nicholls RS, Barrera SM. Haplotypes associated with resistance to sulfadoxine-pyrimethamine in Plasmodium falciparum in two malaria endemic locations in Colombia. Infect Genet Evol. 2013;18:183-90.

41. Mayxay M, Nair S, Sudimack D, Imwong M, Tanomsing N, Pongvongsa T, et al. Combined molecular and clinical assessment of Plasmodium falciparum antimalarial drug resistance in the Lao People's Democratic Republic (Laos). Am J Trop Med Hyg. 2007;77:36-43.

42. Nair S, Miller B, Barends M, Jaidee A, Patel J, Mayxay M, et al. Adaptive copy number evolution in malaria parasites. PLoS Genet. 2008:4:e1000243.

43. Toma H, Imada Y, Vannachone B, Miyagi M, Kobayashi J, Uechi G, et al. A molecular epidemiologic study of point mutations for pyrimethaminesulfadoxine resistance of Plasmodium falciparum isolates from Lao PDR. Southeast Asian J Trop Med Public Health. 2005;36:602-4. 